\title{
Gene Electrotransfer of Canine Interleukin 12 into Canine Melanoma Cell Lines
}

\author{
Ursa Lampreht ${ }^{1} \cdot$ Urska Kamensek $^{1} \cdot$ Monika Stimac $^{1} \cdot$ Gregor Sersa $^{1}$ • \\ Natasa Tozon ${ }^{2} \cdot$ Masa Bosnjak $^{1} \cdot$ Andreja Brozic $^{3} \cdot$ Geraldo Gileno de Sá Oliveira $^{4}$. \\ Takayuki Nakagawa $^{5} \cdot$ Kohei Saeki $^{5} \cdot$ Maja Cemazar $^{1,6}$
}

Received: 13 January 2015/Accepted: 26 March 2015/Published online: 4 April 2015

(C) Springer Science+Business Media New York 2015

\begin{abstract}
A gene electrotransfer (GET) of interleukin 12 (IL-12) had already given good results when treating tumors in human and veterinary clinical trials. So far, plasmids used in veterinary clinical studies encoded a human or a feline IL-12 and an ampicillin resistance gene, which is not recommended by the regulatory agencies to be used in clinical trials. Therefore, the aim of the current study was to construct the plasmid encoding a canine IL-12 with kanamycin antibiotic resistance gene that could be used in veterinary clinical oncology. The validation of the newly constructed plasmid was carried out on canine malignant melanoma cells, which have not been used in GET studies so far, and on human malignant melanoma cells. Canine and human malignant melanoma cell lines were transfected with plasmid encoding enhanced green fluorescence
\end{abstract}

Maja Cemazar

mcemazar@onko-i.si

1 Department of Experimental Oncology, Institute of Oncology Ljubljana, Zaloska 2, 1000 Ljubljana, Slovenia

2 Small Animal Clinic, Veterinary Faculty, University of Ljubljana, Cesta v mestni log 47, Ljubljana, Slovenia

3 Department of Cytopathology, Institute of Oncology Ljubljana, Zaloska 2, 1000 Ljubljana, Slovenia

4 Laboratory of Pathology and Bio-Intervention, Goncalo Moniz Research Center, Oswaldo Cruz Foundation, National Institute of Science and Technology of Tropical Diseases (INCT-DT), Rua Waldemar Falcão, No. 121 Candeal, Salvador, Brazil

5 Laboratory of Veterinary Surgery, Graduate School of Agricultural and Life sciences, The University of Tokyo, 1-1-1 Yayoi, Bunkyo-ku, Tokyo 113-8657, Japan

6 Faculty of Health Sciences, University of Primorska, Polje 42, Izola, Slovenia protein at different pulse parameter conditions to determine the transfection efficiency and cell survival. The IL-12 expression of the most suitable conditions for GET of the plasmid encoding canine IL-12 was determined at mRNA level by the qRT-PCR and at protein level with the ELISpot assay. The obtained results showed that the newly constructed plasmid encoding canine IL-12 had similar or even higher expression capacity than the plasmid encoding human IL-12. Therefore, it represents a promising therapeutic plasmid for further IL-12 gene therapy in clinical studies for spontaneous canine tumors. Additionally, it also meets the main regulatory agencies' (FDA and EMA) criteria.

Keywords Canine IL-12 - Canine melanoma cell lines . Electroporation · Gene electrotransfer - Kanamycin . Plasmid DNA

\section{Introduction}

Murine models have been widely used in cancer research to elucidate the pathways involved in cancer initiation, promotion, and progression (Ranieri et al. 2013). However, due to differences in size and physiology as well as tumor homology between mice and humans, the use of murine cancer models in translational medicine is limited (Kung 2007). Therefore, there is a constant search for new innovative models that are closer to humans. In pet population, cancer is a spontaneous disease and especially dogs develop cancers that share many characteristics with human malignancies (Ranieri et al. 2013). Specifically, dog melanomas develop in the same locations as in humans and they are also homologous to some rare human morphological melanoma types (Gillard et al. 2014). 
Among new biological therapies for melanoma, the IL12 gene therapy showed positive results in the treatment of human melanoma and in canine mastocytoma (Daud et al. 2008; Pavlin et al. 2011). In both studies, gene electrotransfer (GET) was used to facilitate the delivery of plasmid into the target cells. Furthermore, GET of IL-12 was combined with electrochemotherapy in various tumors in dogs (Cutrera et al. 2008; Reed et al. 2010). Electrochemotherapy is another medical application of electroporation, where electrotransfer of chemotherapeutic drugs is used to increase their delivery into tumor cells, thus potentiating their antitumor effectiveness. Electrochemotherapy is a well-established treatment in humans as well as in veterinary oncology (Mali et al. 2013; Scelsi et al. 2013; Tozon et al. 2014). In gene therapy studies using IL-12 in veterinary oncology, either human or feline IL-12 were used as a therapeutic gene due to the nonavailability of canine IL-12 and high homology between canine and human or feline cytokines (86 and $82 \%$, respectively) (Buttner et al. 1998). However, to minimize the possibility of antibody production directed to the heterologous protein that could limit the efficiency of the treatment, there is a need for a therapeutic plasmid encoding canine IL-12. Another concern for safe gene therapy is the presence of antibiotic resistance gene. Namely, according to the U.S. Food and Drug Administration (FDA) and European Medicine Agency (EMA) safety recommendations, only the plasmids encoding genes for resistance against the antibiotics that are not widely used in human or veterinary medicine are allowed in the plasmid vectors intended for the usage in human or veterinary medicine. Currently, only kanamycin resistance gene meets these requirements (FDA 1996; EMA 2011).

Therefore, the aim of the current study was to construct a plasmid encoding IL-12 suitable for gene therapy in veterinary clinical oncology. For this purpose, we constructed a plasmid carrying the sequence for canine IL-12 (dos Santos et al. 2004) and kanamycin antibiotic resistance in order to comply with directives of regulatory agencies. Its activity was evaluated in vitro using GET in canine melanoma cell lines (Inoue et al. 2004) that have not been previously used in GET protocols and in human malignant melanoma cell line.

\section{Materials and Methods}

\section{Construction of Plasmid Encoding Canine IL-12}

The coding sequence for canine IL-12 as a single-chain fusion protein (dos Santos et al. 2004) was cut out of the pcDNA 3.1.ZeosccalL12 plasmid with ApaI and NheI restriction enzymes (Fermentas, Waltham, MA, USA) and cloned directly into a pVax vector (Life Technologies, Grand Island, NY, USA) using a T4 ligase (Fermentas). Escherichia coli strain JM107 (Thermo Scientific Molecular Biology, Vilnus, Lithuania) was transformed with prepared ligation mixture using a TransformAid Bacterial Transformation kit (Thermo Scientific Molecular Biology). Plasmids encoding enhanced green fluorescent protein (EGFP) pEGFP-N1 (Invitrogen, Life Technologies) and human IL-12 pORFhIL12 (Invivogen, Toulouse, France) were used as a positive control. For experiments, all plasmids were purified using Jetstar Plasmid Mega Prep isolation kit (Genomed $\mathrm{GmbH}$, Lohne, Germany) at a concentration of $1 \mathrm{mg} / \mathrm{ml}$. Before each experiment, the identity of each plasmid was verified by restriction analysis. Furthermore, the concentration and purity of each plasmid were verified by spectrophotometry (Epoch Microplate Spectrophotometer, Take $3^{\mathrm{TM}}$ Micro-Volume Plate, BioTek, Bad Friedrichshall, Germany). The ratio of absorbance at 260 and $280 \mathrm{~nm}$ was used to assess the purity of DNA. Only the plasmids with a A260/280 ratio above 1.8 were used in experiments.

\section{Cell Lines}

All cells were cultured in a humidified atmosphere with $5 \% \mathrm{CO}_{2}$ at $37^{\circ} \mathrm{C}$. Canine malignant melanoma CMeC-1 and $\mathrm{CMeC}-2$ cell lines (Inoue et al. 2004) were cultured in Dulbecco modified Eagle medium (DMEM, Life Technologies), and the human malignant melanoma cell line SK-Mel-28 (American Type Culture Collection, Manassas, VA, USA) was cultured in Advanced Minimum Essential Medium (AMEM, Life Technologies). Both media were supplemented with $5 \%$ fetal bovine serum (FBS, Life Technologies), $10 \mathrm{mM} / \mathrm{l}$ L-glutamine (Life Technologies), $100 \mathrm{U} / \mathrm{ml}$ penicillin (Grünenthal, Aachen, Germany), and $50 \mathrm{mg} / \mathrm{ml}$ gentamicin (Krka, Novo Mesto, Slovenia). The doubling time for $\mathrm{CMeC}-1$ cells was $12.7 \pm 1.4$, $15.2 \pm 0.6 \mathrm{~h}$ for $\mathrm{CMeC}-2$ cells, and $24.5 \pm 2.8 \mathrm{~h}$ for SKMel-28 cells.

\section{In Vitro GET}

GET was performed as described previously (Tesic and Cemazar 2013). Briefly, CMeC-1, CMeC-2, and SK-Mel28 cells in exponential growth phase were trypsinized and prepared in ice-cold EP buffer $\left(2.5 \times 10^{7} \mathrm{cells} / \mathrm{ml}\right)$. Aliquots of cells were mixed with different plasmids (pEGFP$\mathrm{N} 1$, pORFhIL12, pCMVcaIL12) at a ratio of 4:1. $50 \mu \mathrm{l}$ of the resulted suspension was pipetted between two parallel stainless steel electrodes with a 2-mm gap in between. GET of plasmids was performed with eight square wave electric pulses with pulse duration of $5 \mathrm{~ms}$ and at a frequency of $1 \mathrm{~Hz}$, generated by the electric pulse generator GT-01 
(Faculty of Electrical Engineering, University of Ljubljana, Slovenia). To determine the optimal voltage for GET of therapeutic plasmids, the cells were exposed to electric pulses in the presence of pEGFP-N1 at voltage-to-distance ratio of $450,500,550$, and $600 \mathrm{~V} / \mathrm{cm}$. Based on the obtain results, all further experiments using pORFhIL12 and pCMVcaIL12 plasmids were carried on at $500 \mathrm{~V} / \mathrm{cm}$ for CMeC- 1 and $\mathrm{CMeC}-2$ cells and at $450 \mathrm{~V} / \mathrm{cm}$ for SK-Mel28 cells. After the electroporation, the cells were incubated for $5 \mathrm{~min}$ in $100 \mu \mathrm{L}$ of FBS and then plated for further assays.

\section{Viability Assay}

After the addition of different plasmids or endotoxin-free water with or without $\mathrm{EP}, 1.0 \times 10^{3} \mathrm{CMeC}-1$ and $\mathrm{CMeC}-2$ and $2.0 \times 10^{3}$ SK-Mel-28 cells were plated in $0.1 \mathrm{ml}$ of appropriate media in 96-well plates (Coring Incorporated, Corning, NY, USA) and incubated at $37{ }^{\circ} \mathrm{C}$ and $5 \% \mathrm{CO}_{2}$ in a humidified incubator. To determine cell viability, Presto-Blue assay (Life Technologies) was performed on day 2 after GET. The fluorescence intensity of the PrestoBlue was measured by a microplate reader (Infinite 200, Tecan, Männedorf, Switzerland). The results were normalized for each group to the viability of control cells. The experiments were repeated twice in triplicates.

\section{Determination of EGFP Expression}

EGFP expression was determined using flow cytometry. Cells were observed under fluorescence microscopy (Olympus IX-70, Hamburg, Germany; excitation 460-495 nm and emission 510-550 nm) at a $\times 10$ objective magnification, 2 days after GET of pEGFP-N1. Their images were captured by a digital color camera (Olympus DP72 CCD) connected to the microscope. After that, the cells were trypsinized, centrifuged for $5 \mathrm{~min}$ at $25{ }^{\circ} \mathrm{C}$ and $1500 \mathrm{rpm}$, resuspended in $400 \mu \mathrm{L}$ of phosphate buffered saline (PBS), and transferred to polystyrene round-bottom tubes (BD Biosciences, San Jose, CA, USA). The percentage of transfected cells, expressing the EGFP and average intensity of the fluorescence of the transfected cells for each experimental group, was determined by FACSCanto II flow cytometer (BD Biosciences). For the excitation and detection of EGFP fluorescence, a 488-nm laser (air-cooled, $20 \mathrm{~mW}$ solid state) and 530/30-nm bandpass filter were used, respectively. To eliminate debris, 20,000 cells were first gated, and afterward a histogram of gated cells against their fluorescence intensity was recorded (software: BD FACSDiva V6.1.2). All of the experiments were repeated twice in duplicates.

\section{IL-12 Expression}

IL-12, mRNA, and protein expression were determined in transfected malignant melanoma cells by quantitative reverse transcription polymerase chain reaction (qRT-PCR) and with ELISpot, respectively. Both assays were performed 2 days after in vitro GET, because the maximum protein expression is the highest from day 2 until 5 days after GET (Cemazar et al. 2004).

For qRT-PCR assay, total RNA was extracted with TRIzol Plus RNA Purification System (Life Technologies). Concentration and the purity of RNA were determined spectrophotometrically (Epoch Microplate Spectrophotometer, Take $3^{\mathrm{TM}}$ Micro-Volume Plate). Transcription of extracted RNA into cDNA was then performed on $500 \mathrm{ng}$ of total RNA extract using SuperScript VILO cDNA Synthesis KIT (Life Technologies). The 10x diluted mixtures of transcribed cDNA were used as a template for the qPCR using SYBR ${ }^{\circledR}$ Green Real-Time PCR Master Mix (Life technologies) that contained primers, SyberGreen (Invitrogen, Life Technologies), and DEPC $\mathrm{H}_{2} \mathrm{O}$ (Ambion, Life Technologies). Primers were designed using IDT primer quest software (Integrated DNA Technologies, Coralville, IA, USA). The best primer combination was selected based on the amplicon length, Tm, and \% GC. Hypoxanthine-guanine phosphoribosyltransferase (HPRT) was used as a reference gene (forward: $5^{\prime}$ TTG TTG TAG GAT ATG CCC TTG AC $3^{\prime}$; reverse: $5^{\prime}$ TTC CAA ACT CAA CTT GAA CTC TCA $3^{\prime}$ ) and IL-12 as a target gene (forward: $5^{\prime}$ CAG GCC CTG AAT AAC AG $3^{\prime}$, reverse: $5^{\prime}$ GCA TGA AGA AGT ATG CAG AGC $3^{\prime}$ ). qPCR was performed on a 7300 System (Applied Biosystem). The thermal cycle protocol consisted of activation of UracilDNA Glycosylase $\left(2 \mathrm{~min}\right.$ at $\left.50{ }^{\circ} \mathrm{C}\right)$, hot start activation of AmpliTaq Gold Enzyme $\left(10 \mathrm{~min}\right.$ at $\left.95{ }^{\circ} \mathrm{C}\right), 45$ cycles of denaturation $\left(15 \mathrm{~s}\right.$ at $\left.95^{\circ} \mathrm{C}\right)$, annealing, and extension $\left(1 \mathrm{~min}\right.$ at $60^{\circ} \mathrm{C}$ ). The 7300 System SDS software (Applied Biosystems) was used for qPCR product analysis. Relative quantification of the qPCR data was performed using $2-\Delta \Delta \mathrm{Ct}$ method (Livak and Schmittgen 2001).

Two days after GET, cells were plated on ELISpot 96-well plates that were coated with antibodies (Canine IL12/IL-23 p40, Human IL-12/IL-23 p40, R\&D systems, Minneapolis, MN, USA). After $4 \mathrm{~h}$ of incubation, cells were removed and the produced canine and human IL-12 were detected by quantitative sandwich enzyme-linked immunosorbent assay technique. Individual wells were imaged by Zeiss SteREO Lumar.V12 (Zeiss, Jena, Germany) stereomicroscope equipped with a MRc.5 digital camera (Zeiss) and spots, representing individual IL-12 secreting cell, were counted. 


\section{Statistical Analysis}

Statistical analysis was performed using Sigma Plot software (Systat software, London, UK). Significance was determined by Holm-Sidak method after one-way analysis of variance (ANOVA) was performed and fulfilled. ${ }^{*} p<0.05$ values were considered as significant. The values were expressed as the arithmetic mean $(\mathrm{AM}) \pm$ standard error of the mean (SEM).

\section{Results and Discussion}

\section{Construction of Plasmid Encoding Canine IL-12}

A plasmid encoding canine IL-12 under the control of CMV promoter with kanamycin antibiotic resistance gene was successfully constructed. Construction of pCMVcaIL12 was verified with ApaI and NheI restriction digestion and electrophoresis (Fig. 1).

In veterinary oncology, published gene therapy studies with IL-12 were performed using plasmid encoding human or feline IL-12 and ampicillin-resistant gene as a bacterial selection gene (Cemazar et al. 2011; Chuang et al. 2009; Cutrera et al. 2008; Pavlin et al. 2011; Reed et al. 2010). Therefore, our newly constructed plasmid holds two advantages in the treatment of canine tumors of clientowned dogs. Firstly, using plasmid encoding canine IL-12, possible allergic reaction of therapy in dogs could be avoided due to complete homology of transfected IL-12 with endogenous.

Secondly, kanamycin is a more appropriate selection marker compared to commonly used ampicillin and it is world wide accepted in human gene therapy clinical trials. In fact, ampicillin is a commonly used broad-spectrum antibiotic in human and veterinary medicine (Solensky 2003); therefore, its use as a selection marker is not acceptable for clinical trials in order to avoid unnecessary risk of antibiotic resistance spread to pathogenic bacteria (FDA 1996; EMA 2011). Furthermore, traces of antibiotics could be found in the final product (e.g., plasmid) which could cause hypersensitivity reactions (Solensky 2003). In addition, the role of antibiotic resistance markers is mainly connected with structural plasmid instabilities and decreased gene delivery efficiency (Oliveira and Mairhofer 2013). So far, only a few studies reported the use of plasmids without the antibiotic resistance gene in preclinical and clinical studies (Spanggaard et al. 2013; Vandermeulen et al. 2011). Therefore, the constructed plasmid pCMVcaIL12 with kanamycin antibiotic resistance and gene encoding canine IL-12 should be suitable and safe for the use in veterinary clinical trials in dogs. (a)

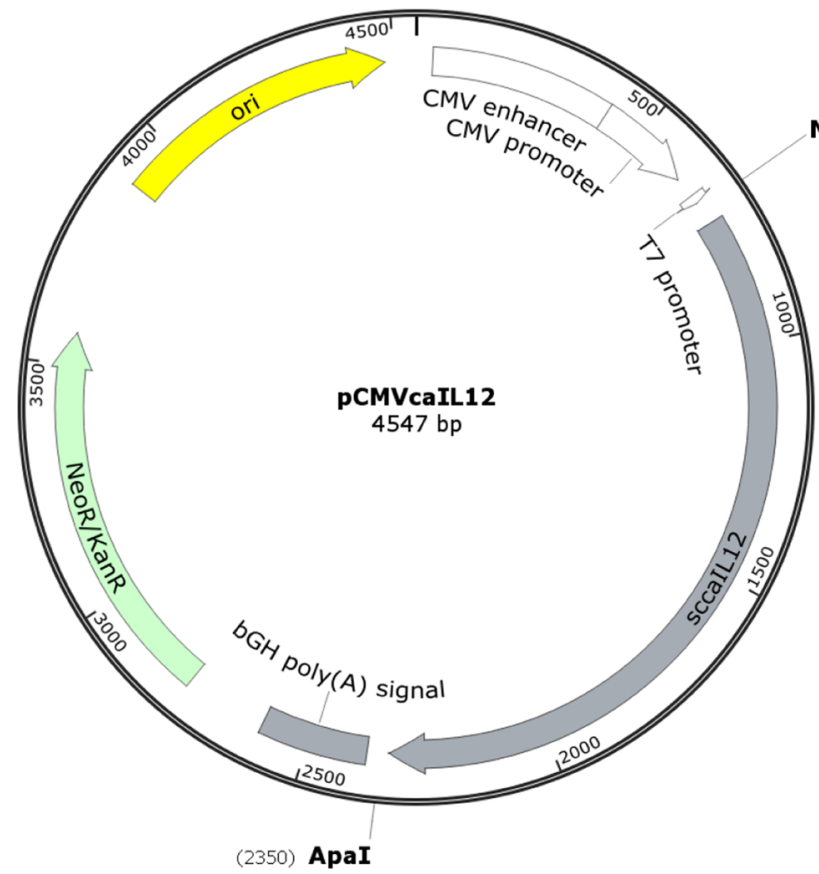

Fig. 1 Restriction analysis confirming the identity of the plasmid. Map of the plasmid (SnapGene) (a), Simulation of the double restriction of the plasmid with ApaI and NheI restriction enzymes (b)

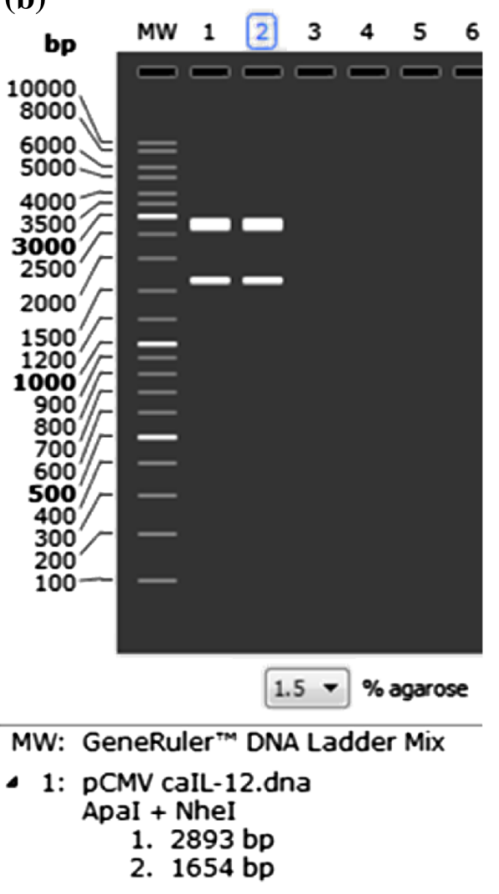

(c)

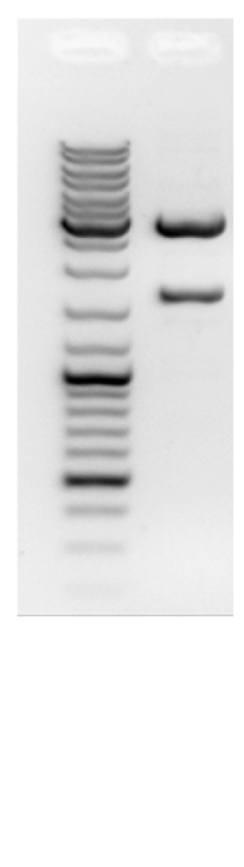

(SnapGene software) (b) and agarose gel image after the electrophoresis of plasmid cut with ApaI and NheI restriction enzymes (c) 


\section{Effect of Different GET Conditions on Transfection Efficiency and Cell Survival in Different Cells}

Several studies demonstrated that the sensitivity of cells varies significantly among different cell lines exposed to the electric pulse (Cemazar et al. 1998; Delteil et al. 2000; Liew et al. 2013; Tesic and Cemazar 2013; Zhou et al. 2012). Therefore, in the current study, the canine melanoma cells (Inoue et al. 2004), which have not been used in GET studies so far, and human melanoma cells were exposed to different GET conditions by varying the amplitude of electric pulses. Both transfection efficiency and survival were measured in order to determine the appropriate GET conditions for further studies.

Transfection efficiency was measured by flow cytometry, determining the percentage of EGFP positive cells, representing the transfection level, and their fluorescence intensity representing the amount of the reporter protein present in the cells, as an indirect measure of the amount of plasmid that was introduced into the cells (Bosnjak et al. 2014). In CMeC-1 cell line, the number of EGFP positive cells was the highest at $500 \mathrm{~V} / \mathrm{cm}$ and was significantly higher compared to the number of positive EGFP cells obtained at $450 \mathrm{~V} / \mathrm{cm}$. Similar results were obtained in CMeC-2 cell line where again $500 \mathrm{~V} / \mathrm{cm}$ showed to be the most appropriate. In human melanoma cell line, SK-Mel-28, the transfection level was the highest at $450 \mathrm{~V} / \mathrm{cm}$. In contrast to the transfection level, the fluorescence intensity per cell in all 3 cell lines did not differ significantly in all four tested conditions and therefore did not have an effect on our selection of the appropriate GET conditions for further experiments (Fig. 2).

Besides the transfection efficiency, another important factor for cells' transfection is their survival following transfection. It is desirable to achieve high transfection efficiency, while retaining cell viability (Dean 2013). In the current study, the survival of cells after GET was determined with a viability assay Presto-Blue. Our results showed that survival of cells, regardless of the cell line tested after GET of pEGFP-N1, was overall the highest at $450 \mathrm{~V} / \mathrm{cm}$ and that survival of $\mathrm{CMeC}-1$ cells was significantly higher than of $\mathrm{CMeC}-2$ and SK-Mel-28 at all tested conditions (Fig. 3a).

Low survival of CMeC-2 and SK-Mel-28 indicated their high sensitivity to GET. High sensitivity was also observed by other researchers in different types of cells, such as normal endothelial (Delteil et al. 2000) and stem cells (Liew et al. 2013), as well as in various cancer cell lines (Tesic and Cemazar 2013; Zhou et al. 2012), not only after GET but also when electroporation was used for enhanced delivery of small molecules into the cells (Cemazar et al. 1998). Increasing voltage at the constant duration of the pulse may increase the transport of macromolecules, but it reduces cell viability that is consequently associated with an overall decrease in transfection efficiency (Rols and Teissie 1998). The pronounced difference in transfection efficiency between different cell types is influenced by many factors. Those factors are both intrinsic, pertinent to the physiology of cells, as well as extrinsic, pertinent to the used GET method (i.e., temperature, buffer composition, parameters of electric pulses, etc.) (Ferreira et al. 2008; Guo et al. 2012; Liew et al. 2013). In the current study, two canine cell lines were used, which derived from the same patient with skin malignant melanoma. The $\mathrm{CMeC}-1$ cells derived from the primary skin melanoma, while the $\mathrm{CMeC}$ 2 cells were isolated from the lung metastasis in nude mice bearing subcutaneous $\mathrm{CMeC}-1$ tumor. Even though the cells originated from the same primary tumor, they exhibited pronounced difference in sensitivity to electroporation, which is most probably due to the phenotypic changes acquired during transplantation to the mouse model (Inoue et al. 2004). CMeC-1 is a highly metastatic cell line, while $\mathrm{CMeC}-2$, although prepared from lung metastasis, does not exhibit metastatic potential. How and whether this difference in metastatic potential influences the sensitivity to GET is currently not known. Hence, there (a)

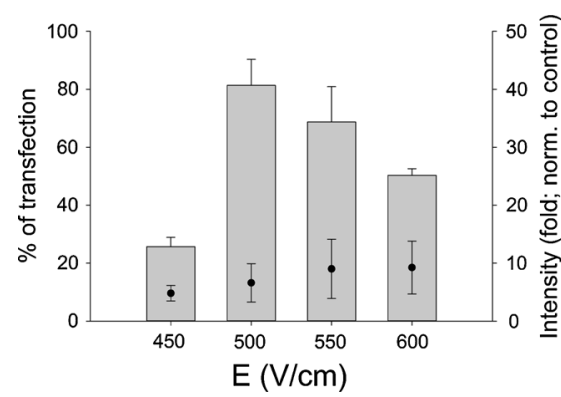

(b)

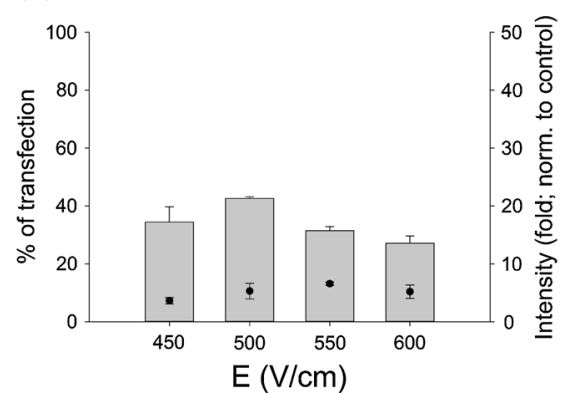

(c)

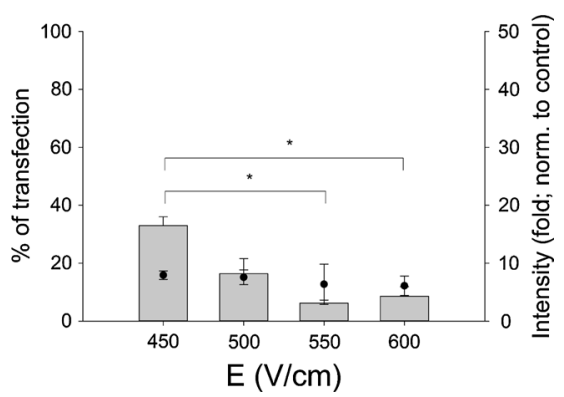

Fig. 2 Transfection level (bar) and median fluorescence intensity (symbol) 2 days after GET of pEGFP-N1 at different GET conditions in two canine malignant melanoma cell lines: $\mathrm{CMeC}-1$ (a) and
CMeC-2 (b); and SK-Mel-28 human malignant melanoma cell line (c). The data are presented as means + SEM. $* p<0.05$ 
(a)

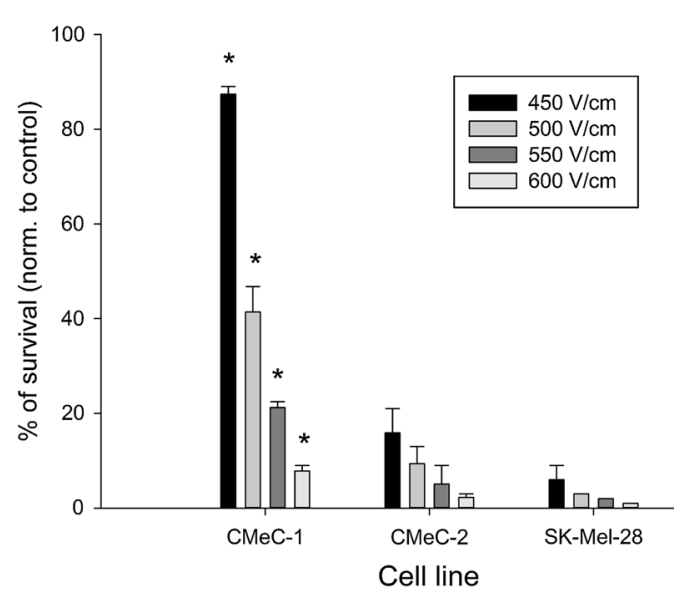

Fig. 3 Survival of the cells on day 2 after transfection with pEGFP$\mathrm{N} 1$ at different GET conditions in two canine melanoma cell lines: CMeC-1 and CMeC-2; and human malignant melanoma SK-Mel-28, measured by Presto-Blue viability assay. Survival is expressed as the percentage of the surviving cells compared to the control cells and is presented as means + SEM. ${ }^{*} p<0.05$ versus CMeC-2 and SK-Mel-

is a strong need for the empirical optimization of GET conditions for each particular cell line. Namely, optimal conditions not only lead to high level of plasmid transferred to the cells, but also minimize cell damage due to the electroporation (Bloquel et al. 2004; Bosnjak et al. 2014). Therefore, based on the results obtained, GET conditions selected for further experiments were $450 \mathrm{~V} / \mathrm{cm}$ for SKMel-28 and $500 \mathrm{~V} / \mathrm{cm}$ for CMeC-1 and CMeC-2 (Fig. 3b).

\section{Effect of GET of Plasmids Encoding IL-12 on Survival and Expression of IL-12 in Different Cells}

In order to evaluate the potential of our newly constructed pCMVcaIL12 for the use in clinical studies, GET of the plasmid was performed in two canine melanoma cell lines and one human melanoma cell line in comparison to GET of pORFhIL12, encoding human IL-12, which has been successfully used in clinical study on canine mastocytoma (Pavlin et al. 2011).

Firstly, cell survival was determined 2 days after GET. The results showed that GET of therapeutic plasmids decreased survival in all three cell lines to the similar level as GET of pEGFP-N1. The survival of cells was the least reduced in $\mathrm{CMeC}-1$ cells and the most in $\mathrm{CMeC}-2$ cells, which was in contrast to the results obtained by pEGFP$\mathrm{N} 1$, where the percentage of survived cells was the lowest in SK-Mel-28 cells. However, these differences were not statistically significant. Importantly, there was no difference in the level of survival with regard to the therapeutic (b)

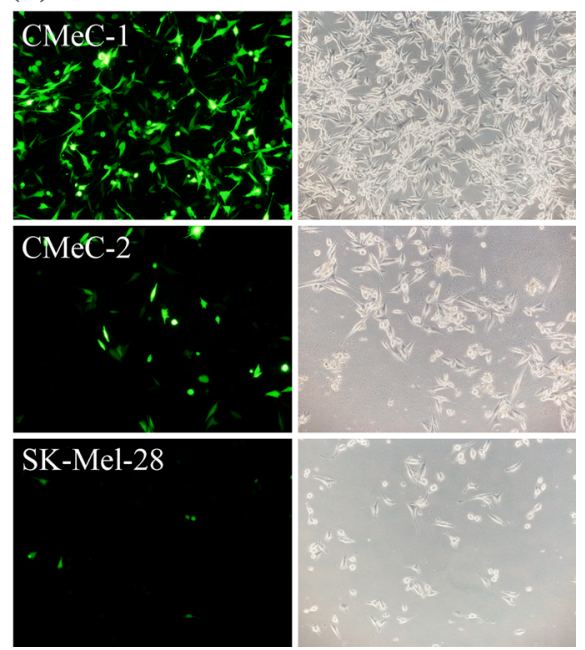

28 at the same conditions and versus other conditions in $\mathrm{CMeC}-1$ (a) transfection efficiency and cell appearances after GET of pEGFP$\mathrm{N} 1$ at optimized GET conditions. The number transfected cells and survival was the highest in CMeC-1 cells and the lowest in SK-Mel28. $\mathrm{CMeC}-1$ and $\mathrm{CMeC}-2$ were exposed to electric pulses at $500 \mathrm{~V} /$ $\mathrm{cm}$ and SK-Mel-28 at $450 \mathrm{~V} / \mathrm{cm}(\mathbf{b})$

plasmid used, demonstrating that the newly constructed plasmid encoding canine IL-12 had the same effect on cell viability as commercial available plasmid encoding human IL-12.

Secondly, the IL-12 expression was determined at mRNA level with the qRT-PCR and at the protein level by ELISpot, where spots represented individual cells secreting IL-12.

The qRT-PCR results demonstrated high expression of IL-12 mRNA after GET of pORFhIL12 and pCMVcaIL12 in both, $\mathrm{CMeC}-1$ and $\mathrm{CMeC}-2$ cell line. The levels of IL-12 mRNA were similar in both $\mathrm{CMeC}$ cell lines and were higher, but non-significant, compared to human SK-Mel-28 (Fig. 4b).

ELISpot results showed a significantly higher number of spots after GET with pCMVcaIL12 compared to GET with pORFhIL12 in all three cell lines. Furthermore, there was also a significant difference in the number of spots between cell lines. $\mathrm{CMeC}-1$ and $\mathrm{CMeC}-2$ had a higher number of spots than SK-Mel-28 (Fig. 4).

As already shown by other studies, the results from mRNA level do not reflect the ability of cells to produce the protein and therefore cannot be used as an indicator for protein level. In general, the half-life of proteins and mRNA are quite different, from around $9 \mathrm{~h}$ for mRNA and up to 2 days for proteins. However, depending on the function of protein, its half-life can vary from minutes to days and therefore the correlation between protein and mRNA half-time could not be drawn (Schwanhausser et al. 2011). The half-life of human IL-12 is estimated to be 
(a)

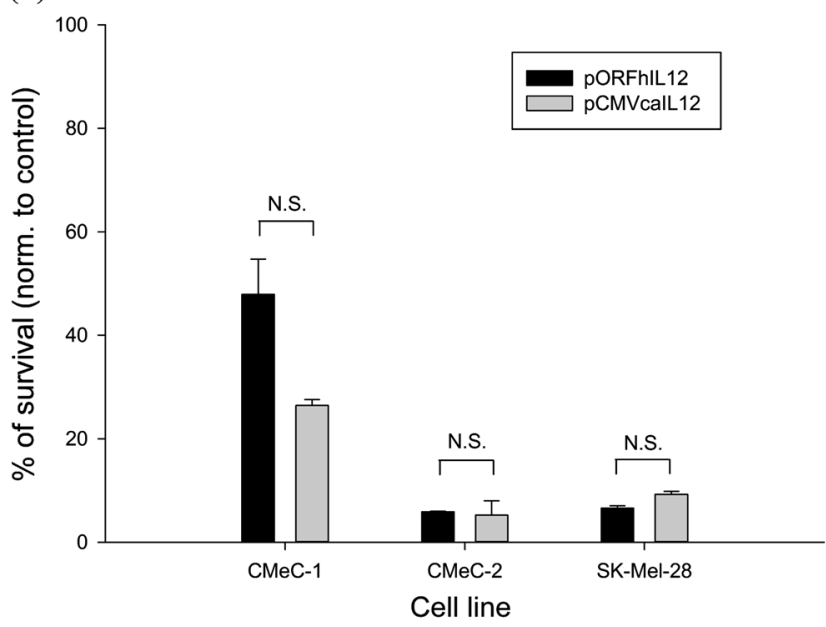

(c)

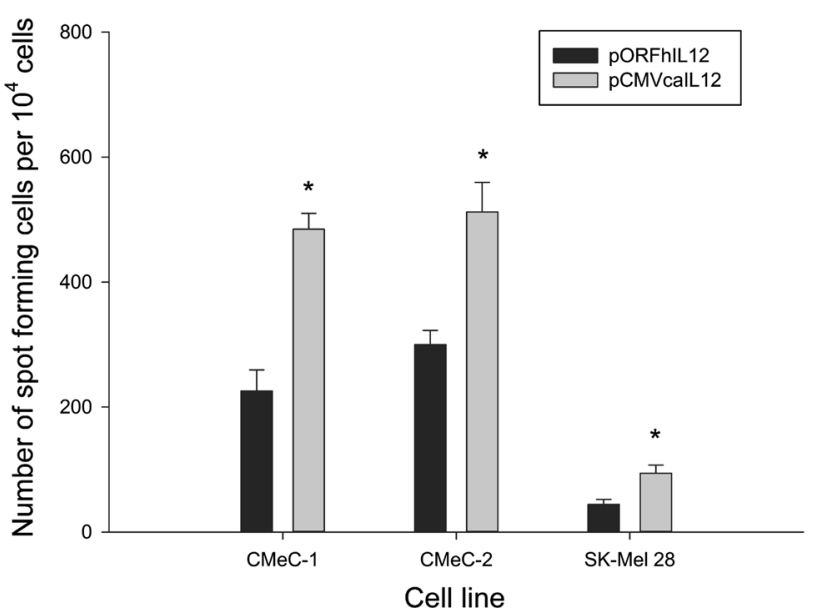

Fig. 4 Survival of cells after GET of pORFhIL12 and pCMVcaIL12 in two canine melanoma cell lines: $\mathrm{CMeC}-1$ and $\mathrm{CMec}-2$; and human malignant melanoma SK-Mel-28. Survival is expressed as the percentage of the surviving cells compared to the control cells and is presented as means + SEM. N.S. represents statistically nonsignificant difference (a) expression of the canine and human IL-12 after transfection with pORFhIL12 and pCMVcaIL12 in two canine melanoma cell lines: $\mathrm{CMeC}-1$ and $\mathrm{CMec}-2$; and human malignant melanoma cell line SK-Mel-28. IL-12 mRNA concentrations are

5-10 $\mathrm{h}$ in serum after intravenous application of recombinant protein, while the data for half-life of IL-12 mRNA are not known, but is presumed to be very short (Atkins et al. 1997).

It is presumed that up to $40 \%$ of protein concentration can be explained by knowing mRNA abundances. Therefore, the expression level of mRNA only explains a fraction of variations in a protein level, but a level of mRNA has been often an excellent predictor for the presence of a protein. It was proposed that mRNA expression may act in a switch-like manner. When the mRNA levels are low, proteins are undetectable, but when the mRNA levels rise, the ability to (b)

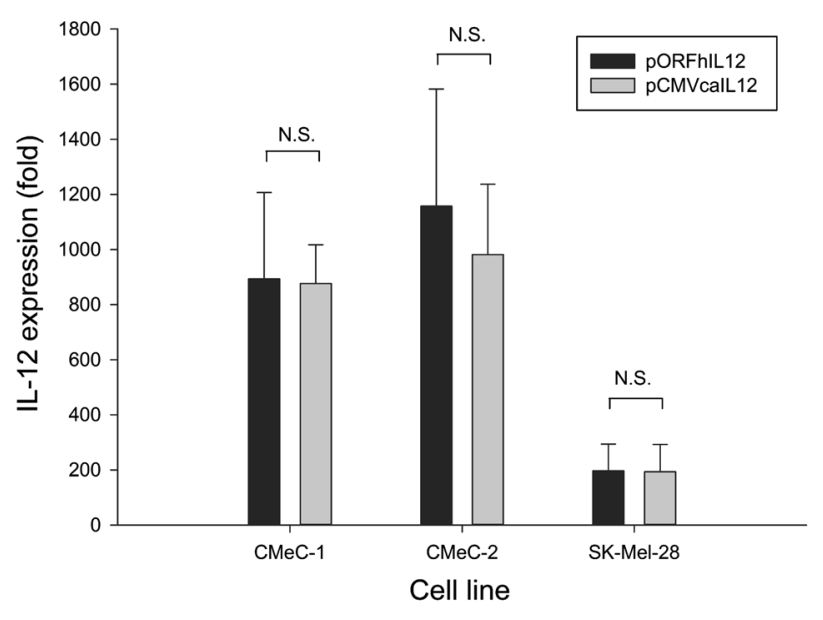

(d)

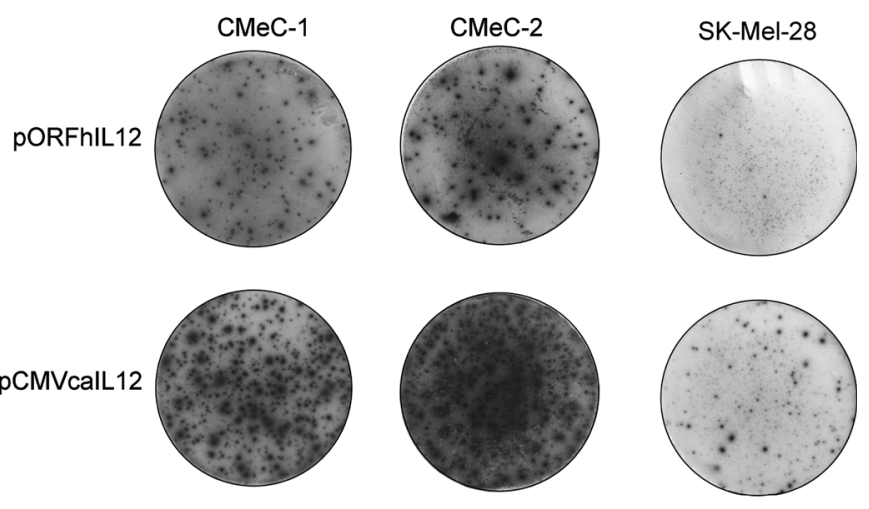

presented as means + SEM. N.S. represents statistically non-significant difference between the plasmids (b) graph showing number of IL-12 forming cells with ELISpot after GET of pORFhIL12 and pCMVcaIL12 in three different cell lines and are presented as means + SEM. The number of spots forming IL-12 was statistically significantly higher $(* p<0.05)$ after GET of pCMVcaIL12 in all three cell lines than after GET of pORFhIL12 (c). Representative images of ELISpot wells showing IL-12 secreting cells 2 days after GET of pORFhIL12 and pCMVcaIL12 (d)

detect proteins increases quickly (Vogel and Marcotte 2012). The correlation between the levels of mRNA and proteins is possible to explore in in vitro cell culture studies. In clinical studies on GET of immunomodulatory genes, the measurement of mRNA levels is difficult because it would require a tissue biopsy. Therefore, alternative approaches are sought, such as the measurement of cytokine levels in the serum or the measurements of mRNA and cytokine level in peripheral blood mononuclear cells. Comparison between the mRNA and protein level of different cytokines in peripheral blood mononuclear cells after vaccination with HPV-16 L1 in human population was done by Shelbl et al. They concluded 
that the degree of correlation between mRNA expression and protein levels varied among different cytokines. The strongest correlation was observed for IFN- $\gamma$, while for IL-12 this comparison could not be performed, because the level of IL12 was below the limit of detection (Shebl et al. 2010).

In conclusion, the results of this study showed that newly constructed plasmid with canine IL-12 and kanamycin antibiotic resistance gene had similar expression of IL-12 compared to plasmid with human IL-12 and ampicillin antibiotic gene, which was previously used in clinical studies in dogs' mastocytomas (Cemazar et al. 2011; Pavlin et al. 2011). Therefore, based on the canine origin, and high expression level demonstrated in the two canine malignant melanoma cell lines, this plasmid represents a promising therapeutic plasmid for further clinical studies with IL-12 GET in spontaneous canine tumors. In addition, the constructed plasmid also meets the criteria of regulatory agencies (FDA 1996; EMA 2011).

Acknowledgments The authors would like to acknowledge M. Lavric for her help with cell cultures. This work was financially supported by the Slovenian Research Agency (programs P3-0003 and P1-0140, Projects J3-4259, J3-6796 and J3-6793). The research was conducted within the scope of LEA EBAM (French-Slovenian European Associated Laboratory: Pulsed Electric Fields Applications in Biology and Medicine) and within the COST TD1104 Action.

\section{References}

Atkins MB, Robertson MJ, Gordon M, Lotze MT, DeCoste M, DuBois JS, Ritz J, Sandler AB, Edington HD, Garzone PD, Mier JW, Canning CM, Battiato L, Tahara H, Sherman ML (1997) Phase I evaluation of intravenous recombinant human interleukin 12 in patients with advanced malignancies. Clin Cancer Res 3:409-417

Bloquel C, Fabre E, Bureau MF, Scherman D (2004) Plasmid DNA electrotransfer for intracellular and secreted proteins expression: new methodological developments and applications. J Gene Med 6(Suppl 1):S11-S23

Bosnjak M, Lorente BC, Pogacar Z, Makovsek V, Cemazar M (2014) Different incubation times of cells after gene electrotransfer in fetal bovine serum affect cell viability, but not transfection efficiency. J Membr Biol 247:421-428

Buttner M, Belke-Louis G, Rziha HJ, McInnes C, Kaaden OR (1998) Detection, cDNA cloning and sequencing of canine interleukin 12. Cytokine 10:241-248

Cemazar M, Jarm T, Miklavcic D, Lebar AM, Ihan A, Kopitar NA, Sersa G (1998) Effect of electric-field intensity on electropermeabilization and electrosensitivity of various tumor-cell lines in vitro. Electromagn Biol Med 17:263-272

Cemazar M, Wilson I, Dachs GU, Tozer GM, Sersa G (2004) Direct visualization of electroporation-assisted in vivo gene delivery to tumors using intravital microscopy — spatial and time dependent distribution. BMC Cancer 4:81

Cemazar, M, Sersa, G, Pavlin, D, Tozon, N (2011) Intramuscular IL-12 electrogene therapy for treatment of spontaneous canine tumors, targets in gene therapy, Prof. Yongping You (Ed.), ISBN: 978-953307-540-2, InTech, doi: 10.5772/20734. http://www.intechopen. com/books/targets-in-gene-therapy/intramuscular-il-12-electrogene- therapy-for-treatment-of-spontaneous-canine-tumors. Accessed 13 Jan 2015

Chuang TF, Lee SC, Liao KW, Hsiao YW, Lo CH, Chiang BL, Lin XZ, Tan MH, Chu RM (2009) Electroporation-mediated IL-12 gene therapy in a transplantable canine cancer model. Int J Cancer 125:698-707

Cutrera J, Torrero M, Shiomitsu K, Mauldin N, Li S (2008) Intratumoral bleomycin and IL-12 electrochemogenetherapy for treating head and neck tumors in dogs. Methods Mol Biol 423:319-325

Daud AI, DeConti RC, Andrews S, Urbas P, Riker AI, Sondak VK, Munster PN, Sullivan DM, Ugen KE, Messina JL, Heller R (2008) Phase I trial of interleukin-12 plasmid electroporation in patients with metastatic melanoma. J Clin Oncol 26:5896-5903

Dean DA (2013) Cell-specific targeting strategies for electroporationmediated gene delivery in cells and animals. J Membr Biol 246:737-744

Delteil C, Teissie J, Rols MP (2000) Effect of serum on in vitro electrically mediated gene delivery and expression in mammalian cells. Biochim Biophys Acta 1467:362-368

dos Santos LR, Barrouin-Melo SM, Chang YF, Olsen J, McDonough SP, Quimby F, dos Santos WL, Pontes-de-Carvalho LC, Oliveira GG (2004) Recombinant single-chain canine interleukin 12 induces interferon gamma mRNA expression in peripheral blood mononuclear cells of dogs with visceral leishmaniasis. Vet Immunol Immunopathol 98:43-48

EMA (European Medicines Agency) (2011) EMA/CAT/GTWP/ 44236/2009: Reflection paper on design modifications of gene therapy medicinal products during development. Retrieved December 20, 2014 from http://www.ema.europa.eu/docs/en GB/document_library/Scientific_guideline/2012/02/WC5001227 43.pdf

FDA (U.S. Food and Drug Administration) (1996) PTC Document Docket No 96-N-0400: Points to consider on plasmid DNA vaccines for preventive infectious disease indications. Retrieved December 20, 2014 from http://www.gpo.gov/fdsys/granule/FR1996-12-27/96-32930

Ferreira E, Potier E, Logeart-Avramoglou D, Salomskaite-Davalgiene S, Mir LM, Petite H (2008) Optimization of a gene electrotransfer method for mesenchymal stem cell transfection. Gene Ther 15:537-544

Gillard M, Cadieu E, De Brito C, Abadie J, Vergier B, Devauchelle P, Degorce F, Dreano S, Primot A, Dorso L, Lagadic M, Galibert F, Hedan B, Galibert MD, Andre C (2014) Naturally occurring melanomas in dogs as models for non-UV pathways of human melanomas. Pigment Cell Melanoma Res 27:90-102

Guo H, Hao R, Wei Y, Sun D, Sun S, Zhang Z (2012) Optimization of electrotransfection conditions of mammalian cells with different biological features. J Membr Biol 245:789-795

Inoue K, Ohashi E, Kadosawa T, Hong SH, Matsunaga S, Mochizuki M, Nishimura R, Sasaki N (2004) Establishment and characterization of four canine melanoma cell lines. J Vet Med Sci 66:1437-1440

Kung AL (2007) Practices and pitfalls of mouse cancer models in drug discovery. Adv Cancer Res 96(96):191-212

Liew A, Andre FM, Lesueur LL, De Menorval MA, O’Brien T, Mir LM (2013) Robust, efficient, and practical electrogene transfer method for human mesenchymal stem cells using square electric pulses. Hum Gene Ther Methods 24:289-297

Livak KJ, Schmittgen TD (2001) Analysis of relative gene expression data using real-time quantitative PCR and the 2(-Delta Delta C(T) ) method. Methods 25:402-408

Mali B, Jarm T, Snoj M, Sersa G, Miklavcic D (2013) Antitumor effectiveness of electrochemotherapy: a systematic review and meta-analysis. Eur J Surg Oncol 39:4-16 
Oliveira PH, Mairhofer J (2013) Marker-free plasmids for biotechnological applications-implications and perspectives. Trends Biotechnol 31(9):539-547

Pavlin D, Cemazar M, Cor A, Sersa G, Pogacnik A, Tozon N (2011) Electrogene therapy with interleukin-12 in canine mast cell tumors. Radiol Oncol 45:31-39

Ranieri G, Gadaleta CD, Patruno R, Zizzo N, Daidone MG, Hansson MG, Paradiso A, Ribatti D (2013) A model of study for human cancer: spontaneous occurring tumors in dogs. Biological features and translation for new anticancer therapies. Crit Rev Oncol Hematol 88:187-197

Reed SD, Fulmer A, Buckholz J, Zhang B, Cutrera J, Shiomitsu K, Li S (2010) Bleomycin/interleukin-12 electrochemogene therapy for treating naturally occurring spontaneous neoplasms in dogs (Retraction of vol 17, pg 571, 2010). Cancer Gene Ther 17:826

Rols MP, Teissie J (1998) Electropermeabilization of mammalian cells to macromolecules: control by pulse duration. Biophys $\mathrm{J}$ 75:1415-1423

Scelsi D, Mevio N, Bertino G, Occhini A, Brazzelli V, Morbini P, Benazzo M (2013) Electrochemotherapy as a new therapeutic strategy in advanced Merkel cell carcinoma of head and neck region. Radiol Oncol 47:366-369

Schwanhausser B, Busse D, Li N, Dittmar G, Schuchhardt J, Wolf J, Chen W, Selbach M (2011) Global quantification of mammalian gene expression control. Nature 473:337-342

Shebl FM, Pinto LA, Garcia-Pineres A, Lempicki R, Williams M, Harro C, Hildesheim A (2010) Comparison of mRNA and protein measures of cytokines following vaccination with human papillomavirus-16 L1 virus-like particles. Cancer Epidemiol Biomark Prev 19:978-981

Solensky R (2003) Hypersensitivity reactions to beta-lactam antibiotics. Clin Rev Allergy Immunol 24:201-220

Spanggaard I, Snoj M, Cavalcanti A, Bouquet C, Sersa G, Robert C, Cemazar M, Dam E, Vasseur B, Attali P, Mir LM, Gehl J (2013) Gene electrotransfer of plasmid antiangiogenic metargidin peptide (AMEP) in disseminated melanoma: safety and efficacy results of a phase I first-in-man study. Hum Gene Ther Clin Dev 24:99-107

Tesic N, Cemazar M (2013) In vitro targeted gene electrotransfer to endothelial cells with plasmid DNA containing human endothelin-1 promoter. J Membr Biol 246:783-791

Tozon N, Pavlin D, Sersa G, Dolinsek T, Cemazar M (2014) Electrochemotherapy with intravenous bleomycin injection: an observational study in superficial squamous cell carcinoma in cats. J Feline Med Surg 16:291-299

Vandermeulen G, Marie C, Scherman D, Preat V (2011) New generation of plasmid backbones devoid of antibiotic resistance marker for gene therapy trials. Mol Ther 19:1942-1949

Vogel C, Marcotte EM (2012) Insights into the regulation of protein abundance from proteomic and transcriptomic analyses. Nat Rev Genet 13:227-232

Zhou W, Xiong Z, Liu Y, Yao C, Li C (2012) Low voltage irreversible electroporation induced apoptosis in $\mathrm{HeLa}$ cells. J Cancer Res Ther 8:80-85 